\title{
Lung perfusion and chest wall configuration is altered by glossopharyngeal breathing
}

\author{
L.M. Seccombe*, S.C.S. Chung*, C.R. Jenkins*, C.J. Frater*, D.W.J. Mackey\#, \\ M.A. Pearson ${ }^{\#}$, L. Emmett ${ }^{\#}$ and M.J. Peters*
}

ABSTRACT: Glossopharyngeal insufflation is used by competitive breath-hold divers to increase lung gas content above baseline total lung capacity (TLC) in order improve performance. Whilst glossopharyngeal insufflation is known to induce hypotension and tachycardia, little is known about the effects on the pulmonary circulation and structural integrity of the thorax.

Six male breath-hold divers were studied. Exhaled lung volumes were measured before and after glossopharyngeal insufflation. On two study days, subjects were studied in the supine position at baseline TLC and after maximal glossopharyngeal insufflation above TLC. Tc $99^{\mathrm{m}}$ labelled macro-aggregated albumin was injected and a computed tomography (CT) scan of the thorax was performed during breath-hold. Single photon emission CT images determined flow and regional deposition. Registered CT images determined change in the volume of the thorax.

CT and perfusion comparisons were possible in four subjects. Lung perfusion was markedly diminished in areas of expanded lung. $69 \%$ of the increase in expired lung volume was via thoracic expansion with a caudal displacement of the diaphragm. One subject who was not proficient at glossopharyngeal insufflation had no change in CT appearance or lung perfusion.

We have demonstrated areas of hyperexpanded, under perfused lung created by glossopharyngeal insufflation above TLC.

KEYWORDS: Breath-hold diving, glossopharyngeal insufflation, hyperinflation, perfusion imaging, pulmonary perfusion

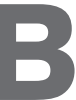
reath-hold diving, or freediving, is a highly organised, increasingly popular extreme sport. Many competitive breathhold divers perform glossopharyngeal breathing both as a training exercise and just prior to a dive or submersed breath-hold. Glossopharyngeal breathing, a pump-like action involving the glossopharyngeal structures and larynx that forces air into the airways [1], was originally developed as a therapeutic technique for neuromuscular patients to help expand tidal volume and cough effectiveness $[2,3]$. The increase in expired lung volume above baseline total lung capacity (TLC) using glossopharyngeal breathing is achieved by a combination of an increase in the Euclidian size of the lung and gas compression [4,5]. Participants refer to this technique as lung packing; however this is described in the literature as glossopharyngeal insufflation (GI) $[1,5,6]$.

In theory, GI above TLC has the potential to assist breath-hold diving performance by increasing available oxygen stores and providing a volume buffer against the compressive effects of hyperbaria. Improvements in both static apnoea duration and breath-hold diving performance have been shown [7]. The potential for adverse cardiocirculatory effects is also clear. The extremely high transpulmonary pressures achieved, of up to $80 \mathrm{cmH}_{2} \mathrm{O}$ [5], are associated with tachycardia, hypotension and biventricular systolic dysfunction [8]. In keeping with these observations, adverse neurological symptoms (e.g. presyncopal episodes and light-headedness) have been associated with this manoeuvre $[6,8]$ and are seen at the peak of GI rather than after a long breath-hold suggesting that these are related to a circulatory effect rather than hypoxia or hypercapnia.

In selected individuals, the increase in expired lung volume above baseline TLC using GI can be as much as $3 \mathrm{~L}[4,5]$. It has been estimated that $\sim 30 \%$ of the additional entrained air can be attributed to gas compression $[4,5]$, with the remainder being an effect of an increase in the Euclidian size of the lung. While some of this increase could be from displacement of structures within the thorax (heart, vessels or oesophagus) most of the increase may be related to the change in the configuration of the chest wall and diaphragm. Individual case reports on breath-hold

\section{AFFILIATIONS}

Depts of *Thoracic Medicine, and

${ }^{\#}$ Nuclear Medicine, Concord

Repatriation General Hospital,

Concord, Sydney, Australia.

CORRESPONDENCE

L.M. Seccombe

Dept of Thoracic Medicine

Concord Repatriation General

Hospital

Hospital Road

Concord

Sydney

NSW 2139

Australia

E-mail: leigh.seccombe@

sswahs.nsw.gov.au

Received:

Oct 142009

Accepted after revision:

Nov 142009

First published online:

Dec 082009 
divers have described pulmonary hyperinflation with cardiac compression, aortic stretch and reduction in blood flow using magnetic resonance imaging $[9,10]$.

In normal lung, there are regional differences in the relationship between alveolar ventilation and capillary blood flow. The under perfusion of apical lung areas is related to a direct effect of gravity on blood flow and an indirect effect of gravitational distortion or lung "sagging" in the normal upright lung [11, 12]. Zone 1, as described by WEST [11], has absent capillary perfusion because alveolar pressure exceeds pulmonary arterial pressure. In healthily individuals these conditions should not exist. If there is a marked increase in alveolar pressure, a significant fall in pulmonary artery pressure or severe distortion of the alveolar capillary bed, zone 1 conditions could exist during GI above baseline TLC.

By any measure, the potential physiological changes during GI at extremely high lung volumes are significant. In this study, we sought to explore the changes in dynamic and regional lung perfusion that occur under these conditions and using computed tomography (CT) imaging asses the accompanying volume changes of lung and thorax in a larger number of subjects than has previously been reported.

\section{METHODS}

Six competitive breath-hold divers who practice GI were recruited. All subjects were nonsmokers and did not have any known lung or heart disease.

\section{Lung function}

Baseline respiratory function was measured according to American Thoracic Society/European Respiratory Society criteria $[13,14]$ using the Vmax Encore (Sensormedics, Yorba Linda, CA, USA). Predicted values were derived from the recommendations of the European Community for Coal and Steel [15]. Exhaled vital capacity (VC) following maximal GI was recorded.

\section{Imaging}

Pulmonary perfusion scintigraphy was performed by intravenous injection of $150 \mathrm{MBq}$ of technetium $99^{\mathrm{m}}$ labelled macroaggregated albumin. A hybrid dual-headed gamma camera (Precedence; Philips Medical Systems, Milpitas, CA, USA) was used to acquire dynamic (0.3 s for 400 frames), planar (eight routine views) and single photon emission computed tomography (SPECT) (15 s at 32 steps per head over an interval of $360^{\circ}$ ) images of the lungs. The same camera (Precedence) acquired a six slice CT of the thorax. For maximum GI above baseline TLC (TLCGI) CT the volume, slice width, interval, pitch and field were $50 \mathrm{~mA}(90 \mathrm{kV}), 5 \mathrm{~mm}, 5 \mathrm{~mm}, 1$ and $35 \mathrm{~cm}$ $\sim 1.1 \mathrm{mSv}$, respectively. For the TLC CT all these measurements were the same with the exception of field which was $25 \mathrm{~cm} \sim 0.8 \mathrm{mSv}$.

Subjects underwent measurements at baseline TLC (TLC) and following TLCGI. These were performed at least $72 \mathrm{~h}$ apart. Following cannulation the subjects lay on a table in the supine position with their arms extended behind their head. Subjects were asked to breath-hold during the dynamic phase of the perfusion imaging ( $\sim 1 \mathrm{~min})$ at TLC or TLCGI. Following this, the subjects immediately underwent static imaging and SPECT imaging ( $\sim 20 \mathrm{~min}$ ) where they breathed normally in the same position.

Low-dose CT scans were performed immediately after perfusion scan completion with subjects replicating the same state as in the perfusion imaging. Subjects were asked to breath-hold for the duration of the CT ( $\sim 80 \mathrm{~s})$.

All imaging was analysed blinded to the subject number, packed or unpacked state and the relative proficiency of subjects in GI.

\section{Processing and display}

Images were analysed independently (Astonish; Philips Medical Systems). For each subject the CT images were registered for direct TLC and TLCGI comparison using crosscorrelation (Syntegra version 2.3.1; Philips Medical Systems) including lung and skeletal tissue. Lung tissue was segmented from the CT images using ITK-SNAP software (University of Pennsylvania, PA, USA) and subsequent analysis was performed using the image processing tool ImageJ (National Institutes of Health, Bethesda, MD, USA).

\section{Imaging interpretation}

SPECT

Images were analysed separately by two nuclear medicine physicians and the average of the two scores were recorded. The analysis was based on a previously reported digitised model of the anatomy of human lung [16]. Each subsegment was graded as either 0 (normal perfusion), 1 (mild perfusion reduction), 2 (moderate perfusion reduction) or 3 (perfusion absent).

\section{Dynamic views}

Time activity curves of the radiotracers were generated. The time between injection and both first detection and maximum counts of radiotracer were recorded for superior vena cava, right upper, middle and lower lobes and left middle lobe.

\section{Computed tomography}

In each subject, TLC Euclidian lung volume was subtracted from TLCGI (from the CT registration) for each CT slice in the transaxial view. The study was reviewed and approved by the Ethics Review Board of the Sydney South West Area Health Service (New South Wales, Australia). Each subject gave written informed consent.

\section{Statistical analysis}

Data are presented as mean $\pm \mathrm{SD}$. A two-tailed paired t-test was used to assess change in physiological parameters between TLC and TLCGI. Inter-observer variability and the comparison of the perfusion intensity scores were performed using the Wilcoxon signed rank test. A p-value $<0.05$ was considered statistically significant.

\section{RESULTS}

The six study subjects were young and healthy. Their demographics, lung function data and breath-hold diving performance history are displayed in table 1. Despite attempting GI, subject 6 was unable to increase his VC with GI on any of three manoeuvres. As planned, this subject proceeded to blinded assessments of perfusion and CT analysis. He functioned 


\begin{tabular}{lcc} 
TABLE 1 & $\begin{array}{l}\text { Baseline lung function and diving performance in } \\
\text { six male breath-hold divers }\end{array}$ \\
Age yrs & $30 \pm 7$ & $20-36$ \\
Height cm & $181 \pm 5$ & $174-190$ \\
BMI $\mathbf{~ k g} \cdot \mathbf{m}^{-2}$ & $25.0 \pm 2.0$ & $22.5-27.4$ \\
FEV $\mathbf{1} \%$ pred & $119 \pm 13$ & $99-132$ \\
FVC L & $6.79 \pm 0.99$ & $5.67-8.62$ \\
FVC \% pred & $129 \pm 16$ & $115-158$ \\
TLC L & $8.42 \pm 1.25$ & $6.81-10.60$ \\
TLC \% pred & $114 \pm 15$ & $97-139$ \\
PB static breath-hold $\mathbf{m i n}$ & $6.33 \pm 1.02$ & $5.30-8.00$ \\
PB dynamic distance $\mathbf{m}$ & $168 \pm 35$ & $135-223$ \\
\hline
\end{tabular}

Data are presented as mean \pm SD or range. BMI: body mass index; FEV1: forced expiratory volume in $1 \mathrm{~s}$; \% pred: \% predicted; FVC: forced vital capacity; TLC: total lung capacity; PB: personal best; dynamic distance: apnoeic distance in a 50-m swimming pool

as a relevant negative control and his results were, therefore, excluded from the analysis of the effect of GI.

All other subjects were able to perform the manoeuvres adequately and were able to increase their measured VC with GI. The mean increase in exhaled VC was $1.4 \pm 0.3 \mathrm{~L}(\mathrm{p}<0.001)$, equivalent to a $20 \pm 3 \%$ increase of baseline VC. CT volume comparisons were not possible in one subject because scanning his greatly increased lung size would have resulted in radiation limits being exceeded. In relation to analysis of lung perfusion, the dynamic views showed that in one subject, the radiotracers did not move into the pulmonary circulation until he had released his breath-hold following TLCGI. His data was not included in the SPECT analysis. Therefore, CT comparisons and perfusion comparisons were each possible in four subjects but the subject sets were different.

\section{Perfusion}

\section{SPECT}

There was good agreement between the observers in the blinded assessment of the change in perfusion intensity between TLC and TLCGI $(\mathrm{p}<0.72)$. In the four subjects that we were able to directly compare, total subsegment scores during TLCGI demonstrated a significant reduction in perfusion intensity from TLC values $(p<0.02)$. There was a reduction in perfusion intensity in anterior $(p<0.03)$ and inferior $(p<0.01)$ segments with no change in posterior, upper and middle segments (figs 1 and 2).

\section{Dynamic views}

Time-activity curves in five subjects demonstrated an increase in time to initial radiotracer activity in the superior vena cava from $10.1 \pm 1.6 \mathrm{~s}$ to $16.3 \pm 14.1 \mathrm{~s}$ from TLC to TLCGI, which did not reach significance. The high variability in the TLCGI time activity was largely attributable the subject who did not experience radiotracer lodgement in the pulmonary microcirculation until release of TLCGI breath-hold. There were no differences in the time to maximum radiotracer counts in the superior vena cava, right upper, middle or lower left lobes or the right middle lobe.

\section{Computed tomography}

In four subjects, Euclidian lung volume (as measured by CT) increased at TLCGI from TLC by $0.97 \pm 0.3 \mathrm{~L}(\mathrm{p}<0.05)$. Areas of expansion were similar across all subjects as demonstrated in
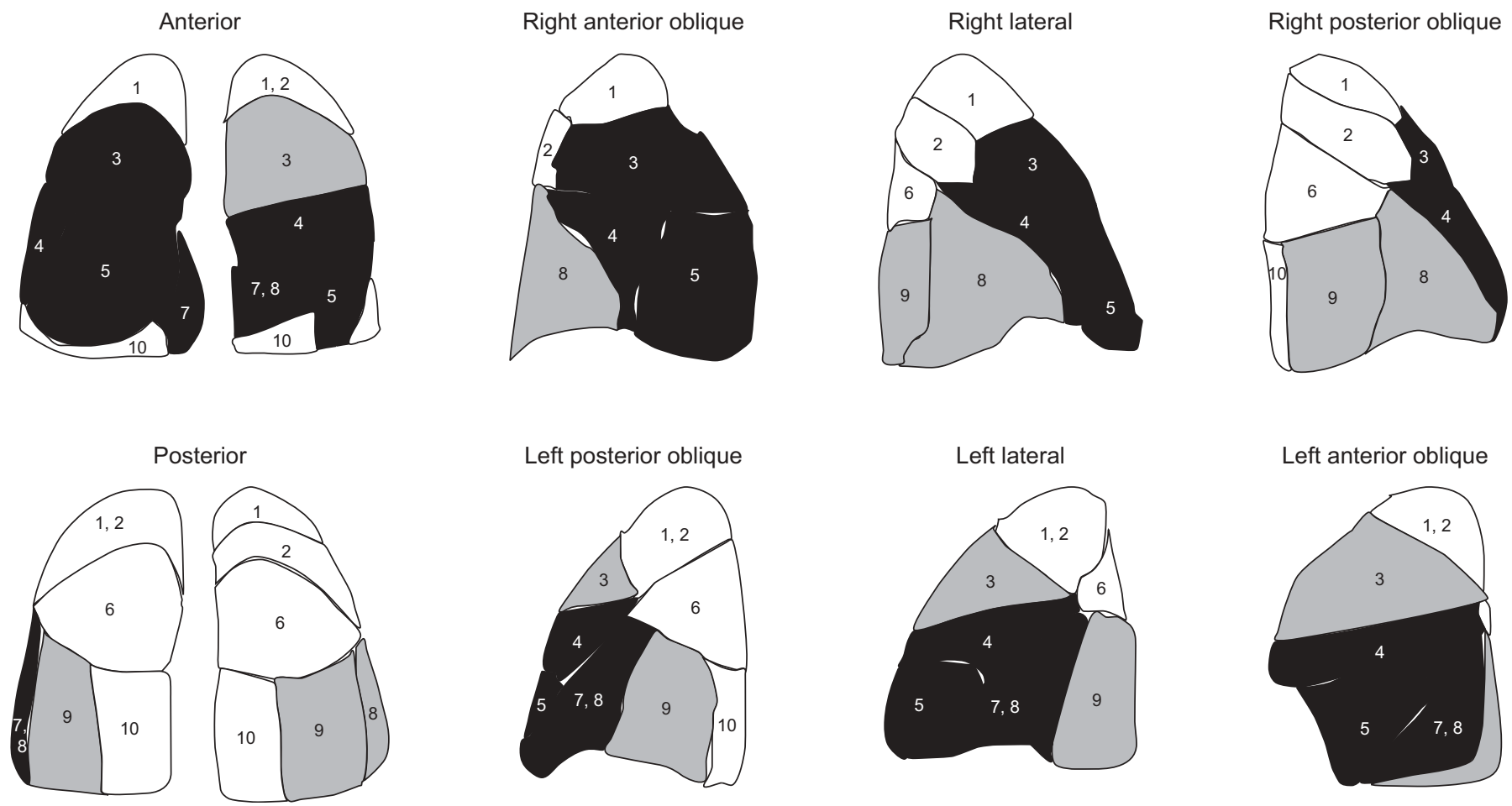

FIGURE 1. Mean reduction in perfusion intensity from baseline to maximum glossopharyngeal insufflation above total lung capacity for each lung subsegment in four breath-hold divers. $\square:<5 \%$ reduction; $\square$ : 6-15\% reduction;

: $16-35 \%$ reduction. The subsegment number as marked according to the Magnussen model [16]. 

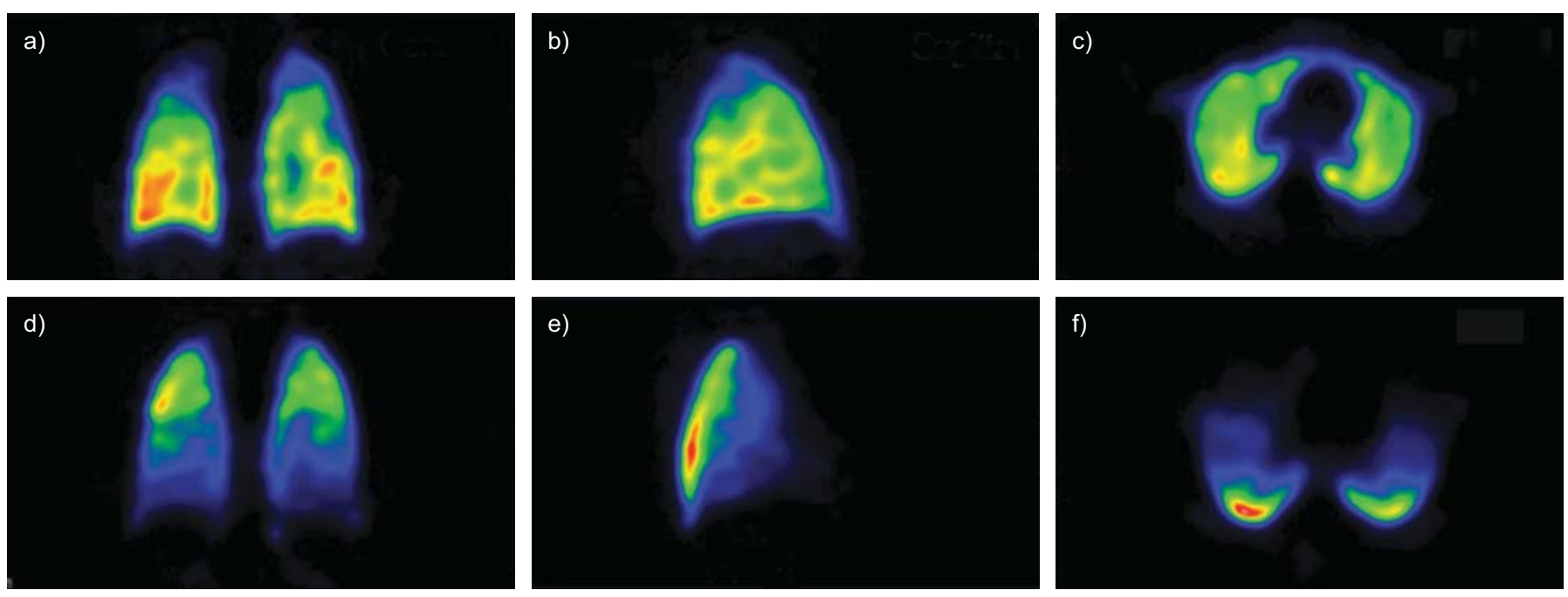

FIGURE 2. Single photon emission computed tomography image of subject 1 in the supine position at a-c) baseline and d-f) at maximal glossopharyngeal insufflation above total lung capacity. a and d) The coronal (posterior) view; b and e) sagittal (right lateral) view; c and f) axial views.

the TLC/TLCGI Euclidian volume subtraction trace for each subject (fig 3). Thoracic expansion was evident with a caudal displacement of the diaphragm (figs 3 and 4). Intercostal bulging of lung tissue and displacement of the vascular mediastinum (fig. 4) was also observed in all subjects. The increase in Euclidian lung volume was $69 \%$ of the increase in VC using expired gas volume measurement. Therefore, gas
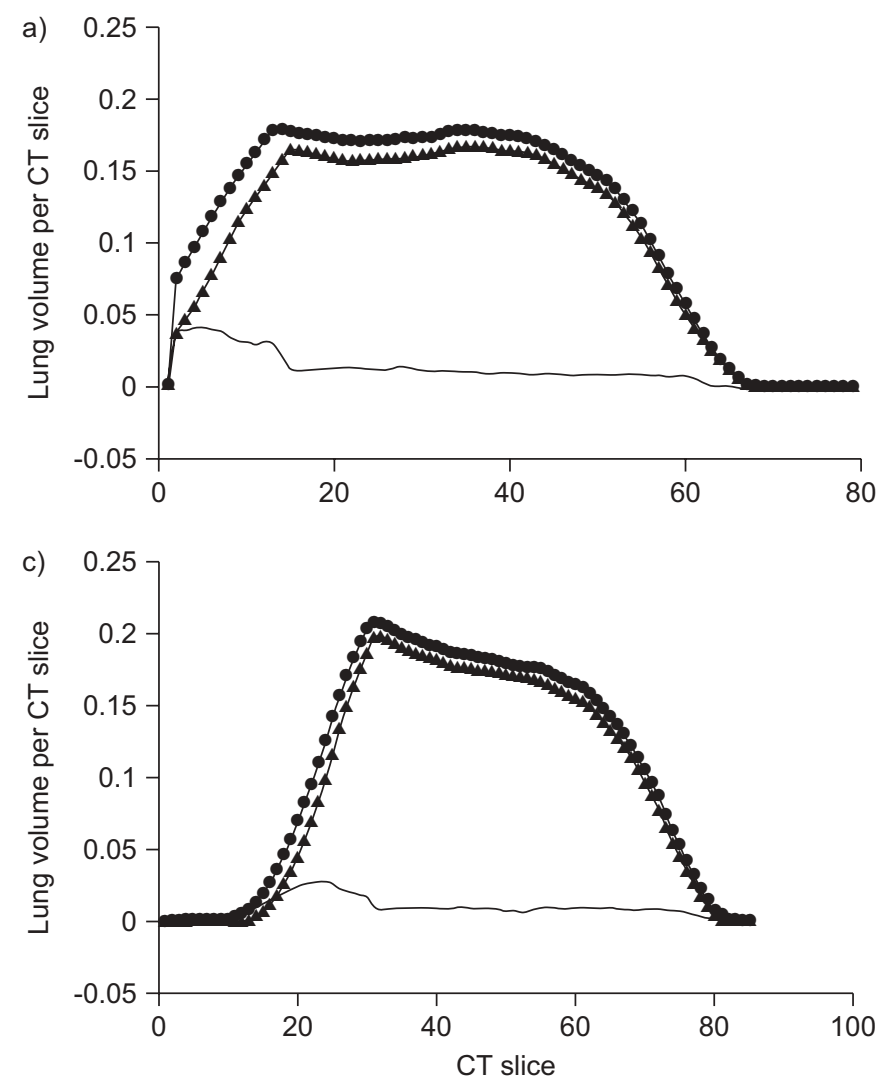

compression was responsible for $\sim 31 \%$ of the increase in expired lung volume after GI.

\section{DISCUSSION}

The study of experienced divers who undertake GI above TLC provides an interesting insight into an area of extreme lung physiology. The increase in lung volume seen in this setting

b)

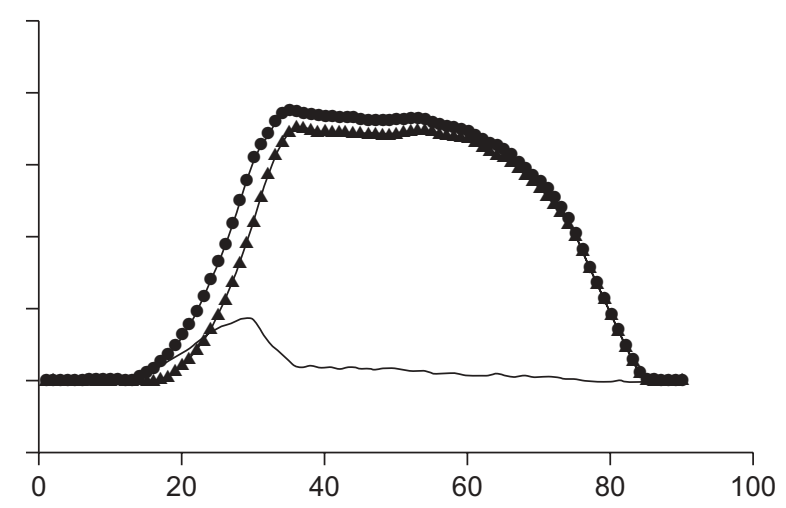

d)

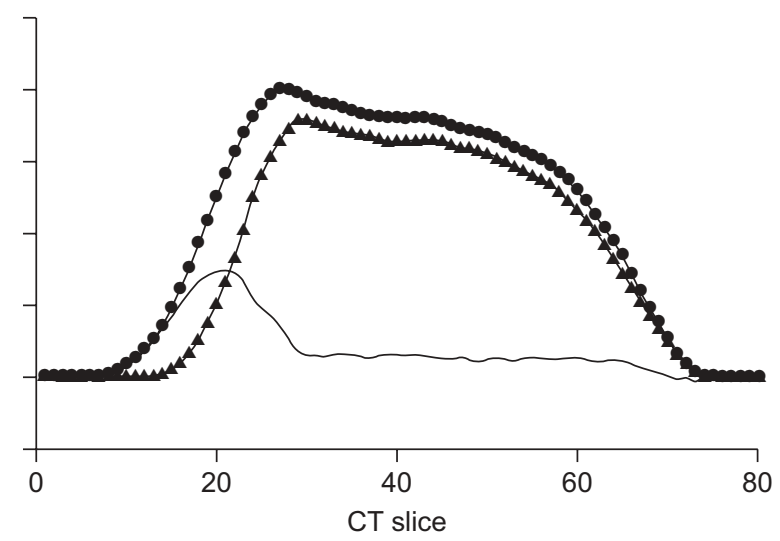

FIGURE 3. Lung volume per computed tomography (CT) slice in the transaxial zone for four breath-hold divers during maximal glossopharyngeal insufflation above total lung capacity $(\bullet)$ and at baseline total lung capacity $(\mathbf{\Lambda})$. a-d) Subjects $2-5$, respectively. The CT slice is numbered from base (0) to apex (>60). ——: subtraction. 


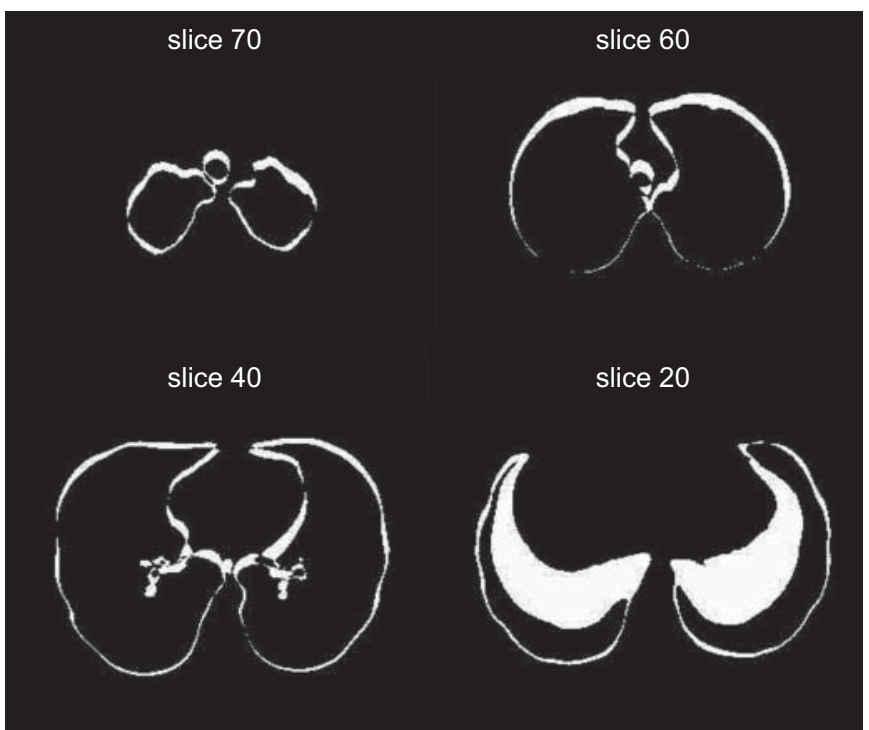

FIGURE 4. Lung volume difference (white areas) between registered computed tomography (CT) scans performed at maximal glossophanyngeal insufflation above total lung capacity (TLC) and baseline TLC in subject 5 . The CT slices displayed are 70 (near apex), 60, 40 and 20 (near base) in the transaxial view.

occurs through thoracic expansion and a downward displacement of the diaphragm along with a displacement of the vascular mediastinum. In the regions of the lung where this marked hyperexpansion occurs there is a reduction in perfusion, approaching absent in some areas.

These subjects had larger than predicted lung size. It is not clear whether this represents a consequence of undertaking GI over a long period or a selection effect that allows competitors with larger lungs than predicted to excel at their chosen sport. By any standard, they were able to increase their expired lung volume remarkably. Using CT-based Euclidian volumetric comparisons, we calculate that $69 \%$ of the additional expired lung volume was achieved through Euclidian lung expansion. Although we did not directly measure alveolar pressure on this occasion, the estimation of the thoracic expansion component of the total increase in expired gas content is identical to the figure estimated in a previous study from alveolar pressure measurement and a pneumotachograph [4] and validates the volumetric calculations conducted herein.

The observed features of lung expansion were that it was achieved through thoracic expansion, a lowering of the diaphragm and displacement of the mediastinum. Reflecting the pressure increase, there was also intercostal bulging of lung tissue. We are confident that our CT registration algorithm was valid and that using anatomical and lung references was appropriate. The consistency of change is reassuring. A previous case report, based on dynamic magnetic resonance imaging, suggested that there was a symmetrical expansion in the thorax [10]. A second case report also demonstrated cardiac and vascular mediastinum configurational changes [9].

Regional differences in lung perfusion can be understood by following three-zone model by WEST [11], which is based on the inequality of blood flow in the lung due to hydrostatic effects. The situation of normal blood flow (zones 2 and 3 ) is where pulmonary arterial pressure is greater than alveolar and pulmonary venous pressures. Zone 1 describes a situation where alveolar pressure becomes greater than pulmonary arterial pressure and, therefore, there is no blood flow. In normal lungs, zone 1 conditions should not exist. The relative lack of perfusion to apical regions in an upright lung is caused by the distortion in the pulmonary capillary bed due to the lung sagging under its own weight within the thorax, not by a gravitational loss of vascular pressure driving perfusion to the apices.

In this study, as the patients were lying in the supine position the gravitational effects had a smaller influence on distribution of lung perfusion than if the subjects had been standing. While there was clearly a vertical gradient to some extent, the axis of the reduction in perfusion was more obliquely placed with a greater reduction in basal, anterior areas than in anterior areas towards the lung apices. Instead of this being entirely gravitational, we believe that the re-distribution must be related to either a marked increase in alveolar pressure in the entire or newly expanded lung and/or an increase in pulmonary vascular resistance in the expanded lung. The latter would be caused by capillary distortion [17].

Previous studies have shown a transient decrease in lung elastic recoil following GI $[4,5,18]$, which is considered to be a result of alveolar stretch associated with repeated and prolonged hyperinflation. Another possible mechanism, as reflected in this data, could be associated with a hyperinflation-related decrease in pulmonary blood content related to capillary distortion, which could, in turn, increase lung compliance. The effect would be similar to that seen with exsanguination [19]. The relatively rapid return of compliance to normal, as has been described previously [18], would be expected as pulmonary capillaries refilled.

One open question is whether the repeated performance of these manoeuvres has a beneficial or deleterious effect on the lung itself. Baseline VC and chest expansion has been shown to significantly increase by $2 \%$ and $10 \%$, respectively, following only 5 weeks GI training in elite female swimmers [20]. We were able to compare static lung function in one subject who participated in an earlier study (table 2) [4]. Over 5 yrs there

\begin{tabular}{lcc}
\hline TABLE 2 & $\begin{array}{l}\text { Baseline lung function in subject } 1 \\
\text { with previous measurements }\end{array}$ \\
& $\mathbf{2 0 0 9}$ & $\mathbf{2 0 0 4}$ \\
\hline Age yrs & 30 & 25 \\
Height $\mathbf{c m}$ & 184 & 186 \\
Weight $\mathbf{~ k g}$ & 89 & 90 \\
FEV $\mathbf{1}$ L & 5.87 & 6.20 \\
FEV $\%$ pred & 129 & 130 \\
FVC L & 8.62 & 8.11 \\
FVC \% pred & 158 & 142 \\
FEV $\mathbf{1}$ FVC $\%$ & 68 & 76 \\
TLC L & 10.60 & 10.08 \\
TLC \% pred & 139 & 130 \\
\hline
\end{tabular}

FEV1: forced expiratory volume in $1 \mathrm{~s}$; \% pred: \% predicted; FVC: forced vita capacity; TLC: total lung capacity. Data for 2004 obtained from [4] 
has been an increase in baseline TLC and VC, which may be performance enhancing but at the expense of a reduction in forced expiratory volume in $1 \mathrm{~s} / \mathrm{VC}$ ratio. In retrospect, the low ratio reported during testing in the earlier study may also have occurred as a result of using this breathing technique in earlier years. The summative effect of these changes on lung performance requires further careful investigation, particularly where this technique has been practiced for many years.

As it might relate to competitive performance in near normobaric conditions (static breath-hold and dynamics within a pool setting), these data could be of interest to breath-hold divers. Presumably, the added gas content achieved by compression is equally distributed to all lung segments. However, the additional entrained air that is added to a poorly perfused lung would contribute little to systemic oxygenation unless there is effective collateral ventilation during and after GI. In addition, it could be predicted that the alveolar pressure of oxygen would fall quite quickly in the perfused lung but remain higher in a lung that less well perfused. To the extent that gravity and body posture influence lung perfusion, changes in posture during face-immersion breath-hold, for example, may lead to greater "extraction" of alveolar oxygen.

There were several methodological and practical challenges associated with our study that are consistent with what is known about this manoeuvre. We discovered that one subject could not successfully use GI to increase his baseline VC and this may occur in a subgroup of competitive breath-hold divers who are uncertain about their competency with this manoeuvre. We also observed syncope in one subject during two separate occasions while attempting TLCGI, which has been previously reported $[6,8]$. Two subjects were studied while breathing tidally for their TLC perfusion scans; however, due to hydrostatic similarities between TLC and tidal breathing we did not believe this to be a limitation.

The subject that was unable to effectively perform GI served as a very useful negative control. There was no change in perfusion intensity or lung shape and the calculated difference in lung volumes between study days was $10 \mathrm{~mL}$. In a frank discussion subsequent to his participation, he admitted that he found no performance benefit from GI and no longer used this manoeuvre when competing. One subject was lost from each of the CT and perfusion comparisons. Whilst we could have studied a greater number of subjects, the evident consistent pattern is such that additional information from more subjects would be unlikely to change our conclusions and the radiation exposure could not be justified. Further studies on these subjects to determine reproducibility of findings were not possible because research radiation limits would have been exceeded.

In summary, dynamic pressure changes in the chest during GI expand lung areas that are aerated but poorly perfused. This primarily affects anterior and inferior regions when subjects are in the supine position. This may be related to increased alveolar pressure, pulmonary capillary pressure decreases or a combination of the two. GI caused thoracic hyperinflation in a pattern that was consistent between subjects.

\section{STATEMENT OF INTEREST}

None declared.

\section{ACKNOWLEDGEMENTS}

We would like to thank Sydney Freedivers (Sydney, Australia) for their support and enthusiasm of our research and their assistance in recruiting subjects. We are also grateful to R. Russo, L. Ridley and P. Rogers for their assistance with data analysis.

\section{REFERENCES}

1 Lindholm P, Norris CM Jr, Braver JM, et al. A fluoroscopic and laryngoscopic study of glossopharyngeal insufflation and exsufflation. Respir Physiol Neurobiol 2009; 167: 189-194.

2 Dail CW, Affeldt JE, Collier CR. Clinical aspects of glossopharyngeal breathing. JAMA 1955; 158: 445-449.

3 Feigelson CI, Dickinson DG, Talner NS, et al. Glossopharyngeal breathing as an aid to the coughing mechanism in the patient with chronic poliomyelitis in a respirator. N Engl J Med 1956; 254: 611-613.

4 Seccombe LM, Rogers PG, Mai N, et al. Features of glossopharyngeal breathing in breath-hold divers. J Appl Physiol 2006; 101: 799-801.

5 Loring SH, O'Donnell CR, Butler JP, et al. Transpulmonary pressures and lung mechanics with glossopharyngeal insufflation and exsufflation beyond normal lung volumes in competitive breath-hold divers. J Appl Physiol 2007; 102: 841-846.

6 Novalija J, Lindholm P, Loring SH, et al. Cardiovascular aspects of glossopharyngeal insufflation and exsufflation. Undersea Hyperb Med 2007; 34: 415-423.

7 Overgaard K, Friis S, Pedersen RB, et al. Influence of lung volume, glossopharyngeal inhalation and $\mathrm{P}(\mathrm{ET}) \mathrm{O}_{2}$ and $\mathrm{P}(\mathrm{ET}) \mathrm{CO}_{2}$ on apnea performance in trained breath-hold divers. Eur J Appl Physiol 2006; 97: 158-164.

8 Potkin R, Cheng V, Siegel R. Effects of glossopharyngeal insufflation on cardiac function: an echocardiographic study in elite breath-hold divers. J Appl Physiol 2007; 103: 823-827.

9 Lindholm P, Nyŕen S. Studies on inspiratory and expiratory glossopharyngeal breathing in breath-hold divers employing magnetic resonance imaging and spirometry. Eur J Appl Physiol 2005; 94: 646-651.

10 Eichinger M, Walterspacher S, Scholz T, et al. Lung hyperinflation: foe or friend? Eur Respir J 2008; 32: 1113-1116.

11 West JB. Regional differences in the lung. Chest 1978; 74: 426-437.

12 Arai TJ, Henderson AC, Dubowitz DJ, et al. Hypoxic pulmonary vasoconstriction does not contribute to pulmonary blood flow heterogeneity in normoxia in normal supine humans. J Appl Physiol 2009; 106: 1057-1064.

13 Miller MR, Hankinson J, Brusasco V, et al. Standardisation of spirometry. Eur Respir J 2005; 26: 319-338.

14 Wanger J, Clausen JL, Coates A, et al. Standardisation of the measurement of lung volumes. Eur Respir J 2005; 26: 511-522.

15 Quanjer PH. European Community for Coal and Steel. Standardized lung function testing. Bull Eur Physiopathol Respir 1983; 19: Suppl. 5, 7-21.

16 Magnussen JS, Chicco P, Palmer AW, et al. Creation of a threedimensional model of human segmental lung anatomy. AJR Am J Roentgenol 2000; 174: 1333-1336.

17 Measurement of pulmonary blood flow. In: West JB, ed. Respiratory Physiology. The essentials. 6th Edn. Baltimore, Lippincott Williams and Wilkins, 2000; p. 35.

18 Tetzlaff K, Scholz T, Walterspacher S, et al. Characteristics of the respiratory mechanical and muscle function of competitive breathhold divers. Eur J Appl Physiol 2008; 103: 469-475.

19 Lung and chest wall elasticity. In: Cotes JE, Chinn DJ, Miller MR, eds. Lung function. 6th Edn. Malden, Blackwell Publishing, 2006; p. 123.

20 Nygren-Bonnier M, Gullstrand L, Klefbeck B, et al. Effects of glossopharyngeal pistoning for lung insufflation in elite swimmers. Med Sci Sports Exerc 2007; 39: 836-841. 